\title{
Molecular behaviors in thin film lubrication-Part one: Film formation for different polarities of molecules
}

\author{
Shaohua ZHANG ${ }^{1,2}$, Yijun QIAO ${ }^{1}$, Yuhong LIU ${ }^{1, *}$, Liran MA ${ }^{1}$, Jianbin LUO ${ }^{1, *}$ \\ ${ }^{1}$ State Key Laboratory of Tribology, Tsinghua University, Beijing 100084, China \\ ${ }^{2}$ Beijing Key Laboratory of Long-life Technology of Precise Rotation and Transmission Mechanisms, Beijing Institute of Control \\ Engineering, Beijing 100094, China
}

Received: 29 August 2018 / Revised: 28 January 2019 / Accepted: 07 March 2019

(C) The author(s) 2019.

\begin{abstract}
There are three unsolved problems in thin film lubrication (TFL) since it was proposed 20 years ago, i.e., the determination of the type of molecules that can enter the contact region efficiently during sliding, the orientation of molecules in the contact region, and the effect of solid surfaces on the liquid molecular orientation in TFL. In order to answer the first two questions, an in situ measurement system comprising a self-designed Raman microscopy and relative optical interference intensity (ROII) system was set up to study the molecular behaviors. A variety of binary mixtures were used as lubricants in the test, and the concentration distribution profile and orientation of the additive molecules in TFL were characterized. The molecular behavior was determined via a combination of shearing, confinement, and surface adsorption. Furthermore, the difference in molecular polarity resulted in different competing effect of surface adsorption and intermolecular interaction, the influence of which on molecular behavior was discussed. Polar additive molecules interacted with the steel surface and exhibited an enrichment effect in the Hertz contact region when added into a nonpolar base oil. No enrichment effect was observed for nonpolar molecules that were added into the nonpolar base oil and polar molecules added into polar base oil. The enrichment of additive molecules enhanced the film-forming ability of the lubricant and resulted in a reduction in the friction coefficient of up to $61 \%$. The orderly arrangement of the additive molecules was another reason for the friction-reducing. A binary multilayer model was proposed to illuminate the molecular behavior in the TFL, and the model was supported by contrary experiment results in elastohydrodynamic lubrication. This research may aid in understanding the nanoscale lubrication mechanism in TFL and the development of novel liquid lubricants.
\end{abstract}

Keywords: thin film lubrication; in situ Raman spectroscopy; molecular distribution; molecular orientation

\section{Introduction}

Molecular orientation has gained increasing interest in the field of electronics [1-3], biology [4-6], surface science [7-11] and tribology in recent years.

The detection and control of molecular orientation aid in the development of novel technologies such as organic solar cells [2], biosensors [6], superhydrophobic surfaces $[7,8]$ and surface inhibitors [9-11]. The molecular stacking and crystallinity significantly influence the exciton dissociation and free charge transport and result in different power conversion efficiencies [3]. The specific control of molecular orientation enables better biomolecule conjugation and improves biocompatibility [4]. The superhydrophobic surface of polyvinyl alcohol nanofibers [7] was obtained owing to the reorientation of hydrophobic backbones at the air-solid interface. Moreover, molecules under confinement were found to exhibit specific phenomena such as reorientation $[12,13]$ and phase transition

* Corresponding authors: Yuhong LIU, E-mail: liuyuhong@tsinghua.edu.cn; Jianbin LUO, E-mail: luojb@tsinghua.edu.cn 


\section{Nomenclature}

TFL Thin film lubrication

EHL Elastohydrodynamic Lubrication
5CB 4-n-pentyl-4'-cyanobiphenyl

$S \quad$ Degree of orientational anisotropy
$[14,15]$. There are mainly three approaches to build a confinement environment, including a pore surface [14-16], microfluidic device [12, 17], and contact region in tribology [13, 18]. In the field of tribology, much attention has been focused on lubricants confined in nano-sized gaps. Itoh et al. [19] studied the orientation parameter and viscosity of $5 \mathrm{CB}$ confined and sheared in a gap of size ranging from $500 \mathrm{~nm}$ to a few $\mathrm{nm}$ using a surface force apparatus (SFA). Zhang et al. [20] explored the molecular orientation and friction performance of $5 \mathrm{CB}$ confined in the contact region with a gap size of tens of nanometers. When the gap size was in the range of several nanometers to tens of nanometers, the lubricant regime was called thin film lubrication (TFL), which was proposed by Luo et al. [21, 22], who obtained measurements with a relative optical interference intensity (ROII) system during 1994 to 1996, wherein the liquid molecule behaviors were found to be related to both the physical and chemical characteristics of the solid surfaces. In the TFL regime, the lubricant film is dominated by the molecular behaviors in different micro/nano regions, which involves three layers - i.e., the adsorbed layer, which is a monomolecular layer wherein molecules are absorbed and packed onto the solid surface; the dynamic fluid layer, which is at the center of the gap; and the ordered layer, which is between the absorbed and fluid layers-being created during the shearing process as the transition region. The lubrication in the ordered layer can be defined as TFL [23, 24].

Since the discovery of the TFL regime, by virtue of the development of new approaches introduced by the flourishing of nanotechnology, the global focus has been on characterizing the TFL in the past twenty decades. Ma and Luo [25] summarized and reviewed the development of TFL in the past 20 years in 2016. Based on the spacer layer interferometry [26], the film formation performances of both surfactant and polymer solutions have been investigated by Spikes et al. [27, 28], while attributing the enhanced film thickness in the TFL regime to the adsorption and packing layers of lubricant molecules on the solid surfaces. They successfully investigated the behaviors of zinc dialkyldithiophosphate (ZDDP) in TFL recently [29]. By utilizing the ROII technique [30, 31], Luo et al. [32] reported a gradually increased film formation with time, which is considered to have been induced by the thickening of the ordered solid-like layer, and the failure condition of TFL. Such positive deviations of the film thickness from the Hamrock-Dowson prediction have been commonly considered to result from the enhanced effective viscosity of the lubricant on the solid surfaces. Thus the packing of the liquid molecules can also be achieved by applying an external electric field to the contacted surfaces. Luo et al. [33] reported an enhanced film thickness at a relatively high electric voltage, which is caused by the rearrangement of polar molecules at the solid surfaces. Thereafter, a similar phenomenon with an increased film thickness has been observed on polar liquid-ionic and aliphatic compound liquid molecules by Xie et al. [34, 35], which was reported as the electric-filed-induced "freezing" of the lubricant when the film thickness is greater than $10 \mathrm{~nm}$ in the TFL regime. Hartl et al. [36, 37] successfully demonstrated the TFL of hexadecane under an extremely high pressure by developing colorimetric interferometry [36]. In 2016, they investigated the lubricant flow in thin-film elastohydrodynamic contact under extreme conditions [38]. All the above achievements benefited from the improved optical interference techniques developed by the above mentioned tribologists. Moreover, similar optical technology developed by Guo et al. [39] and Zhang et al. [40] on the basis of the ROII method [22] can also be used to investigate the TFL characteristics with a resolution of $1 \mathrm{~nm}$.

During the past 20 years, owing to instrumental limitations, several issues still lack effective solutions, for instance, the determination of the types of molecules that can enter the contact region more efficiently during sliding, the orientation of molecules in the contact region, and the effect of solid surfaces on the liquid 
molecular orientation in TFL. With the objective of solving these issues, direct and in situ detection of the lubricant molecules in the contact region has been regarded as the most efficient method of revealing the molecular behaviors, such as molecular ordering, arrangement, orientation and distribution, in the TFL regime. However, it is difficult to measure such in situ molecular signals using conventional methods. Molecular spectrum approaches have been quite effective in detecting lubricant molecular behaviors under shear, e.g., the molecular ordering and orientation, by introducing spectroscopy to the friction systems. Recently, the infrared spectrum [41], Raman spectrum [20, 42-45], sum-frequency generation [46, 47], as well as X-rays [48] have been used to study lubricants in confinement. Owing to the high spatial resolution and sensitivity to molecular orientation of polarized laser light, the Raman spectrum has been used to measure the molecular behaviors in the contact region in several studies. By introducing Raman spectroscopy to the SFA, Bae et al. [46] demonstrated the heterogeneous surface-induced molecular structures. Zhang et al. [20] have succeeded in determining the molecular structure and arrangement of single-phase lubricant molecules in situ. It has been widely proved that the interfacial molecular arrangement is under the effect of shear, surface adsorption, and confinement. However, the internal interaction between components in multiphase lubricants is less studied. To answer the question regarding the type of molecules that can enter the contact region more efficiently during sliding, a systematic study is required.

With the aim of solving the above issue, in the present study, additives and base oils with different polarities were mixed to performed lubrication experiments in the TFL regime by applying a suitable sliding speed and load. Lubricating films in the (elastic-hydrodynamic lubrication) EHL regime were also detected as a reference. The concentration distribution profiles of the additive molecules were mapped over a region of $200 \mu \mathrm{m} \times 200 \mu \mathrm{m}$ using an in situ Raman-tribology system. The center-film thickness and friction coefficient were monitored under the same conditions as those of the Raman experiments. The orientational anisotropy of the additive molecules was measured using polarized Raman spectra. It is revealed how the molecules move and are arranged in the lubricating film at the nanoscale, which contributes to our understanding of the lubricant film formation and failure in the TFL regime [49].

\section{Experiment}

\subsection{Materials}

In the experiments, nematic liquid crystals of 4-n-pentyl4'-cyanobiphenyl (5CB) were purchased from Huarui Scientific and Technological Co. (Hebei, China). Phenethyl alcohol, acetophenone, biphenyl, hexadecane, and decyl alcohol were purchased from Sinopharm Chemical Reagent Co., Ltd. (Beijing, China). All the chemicals, with their initial purity of no less than $99.0 \%$, were used without further purification.

Six mixed lubricants (Lubricants 1, 2, 3, 4, 5, and 6) were prepared with a weight concentration of $10 \%$, except for Lubricant 2, as shown in Table 1. As the dynamic viscosity of phenethyl alcohol $(7.58 \mathrm{mPa} \cdot \mathrm{s})$ is much larger than that of acetophenone $(1.68 \mathrm{mPa} \cdot \mathrm{s})$ and liquid biphenyl (1.48 $\mathrm{mPa} \cdot \mathrm{s})$, the concentration of phenethyl alcohol in Lubricant 2 is $2.5 \%$ in order to obtain a viscosity similar to that of Lubricants 1, 3, and 4 . The additives and base oils in the six mixtures have different polarities and functional groups which are also presented in Table 1.

The above molecules were selected because they have either great potential as lubricants or great significance for our understanding of the molecular behaviors of lubricants. 5CB liquid crystals have great solubility in common base oils because of their similar structure. It has been studied as a lubricant and additive, and shows good potential in reducing friction and externally controlling the lubricating performance [19]. Furthermore, 5CB molecules arrange in an orderly manner under the effect of surface adsorption [17, 19] and flow alignment [12], which makes it an excellent model for studying the molecular orientation of the lubricant. Alkanes with sixteen or more carbon atoms form the most important components of lubricants. Hexadecane is commonly used as a base oil in researches on friction and lubricants. In addition to its stable physical and chemical properties, its nonpolar property is another reason to select hexadecane as the base oil in our research. Functional groups such as hydroxy, benzene ring, and ketone are common in 
Table 1 The composition of six lubricants and their viscosities.

\begin{tabular}{|c|c|c|c|c|c|c|}
\hline & Lubricant 1 & Lubricant 2 & Lubricant 3 & Lubricant 4 & Lubricant 5 & Lubricant 6 \\
\hline \multirow[t]{2}{*}{ Additive } & & & & & & \\
\hline & $5 \mathrm{CB}$ & $\begin{array}{l}\text { Phenethyl } \\
\text { alcohol }\end{array}$ & $\begin{array}{l}\text { Acetophe } \\
\text { none }\end{array}$ & Biphenyl & \multicolumn{2}{|c|}{$5 \mathrm{CB}$} \\
\hline wt $\%$ & $10.0 \%$ & $2.5 \%$ & $10.0 \%$ & $10.0 \%$ & $10.0 \%$ & $10.0 \%$ \\
\hline Raman band of additive $\left(\mathrm{cm}^{-1}\right)$ & 1,607 & 1,007 & 1,002 & 1,004 & 1,607 & 2,227 \\
\hline \multirow[t]{2}{*}{ Base oil } & & & & & 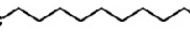 & \\
\hline & \multicolumn{4}{|c|}{ Hexadecane } & Decyl alcohol & Phenethyl alcohol \\
\hline Raman band of base oil $\left(\mathrm{cm}^{-1}\right)$ & \multicolumn{4}{|c|}{2,890} & 2,890 & 1,001 \\
\hline Viscosity $(\mathrm{mPa} \cdot \mathrm{s})$ & 4.55 & 3.64 & 3.30 & 3.65 & 11.90 & 11.34 \\
\hline
\end{tabular}

lubricants. However, little attention has been focused on studying the influence of different functional groups on the distribution and orientation of lubricant molecules. Phenethyl alcohol, acetophenone, biphenyl, and decyl alcohol are selected as lubricants in our research, because they have simple structures and only consist of functional groups that we are concerned with. The second reason to select these molecules is that they have strong Raman signals, which can be distinguished by our in situ Raman-tribology system. It has been proved that the Raman peak of the benzene ring in aromatic compounds and $\mathrm{C}-\mathrm{H}$ in molecules with a long carbon chain can be easily distinguished. Molecules with these two structures, such as 6CB [50], liquid benzene-d6 [51], and n-C16H34 [52] are widely studied using the Raman spectrum. As the resolution of the in situ Raman spectrum is lower than that of the traditional offline Raman, there are higher requirements of signal strength for molecules used in in situ Raman study. The six molecules are selected in our research because they have a benzene ring or $\mathrm{C}-\mathrm{H}$ bond, and they provided a strong signal in the in situ experiments.

\subsection{Molecular distribution measurements}

The concentration distribution profile and dynamic of the confined liquid thin film in the contact region were measured using an in situ Raman-tribology system, as shown in Fig. 1(a). In the tribol ogy part, a circular contact region was formed between a flat silica glass surface and reflective steel ball. The surface roughness of the glass disk and steel ball was less

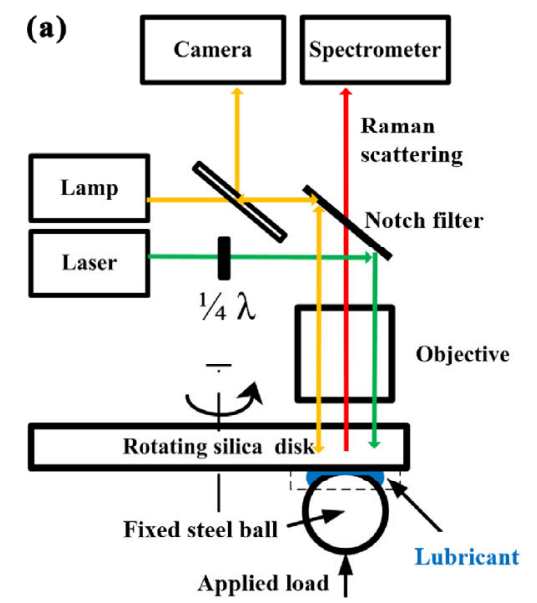

(b)

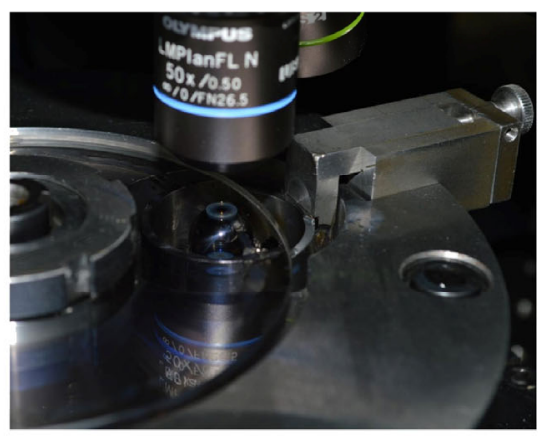

(c)

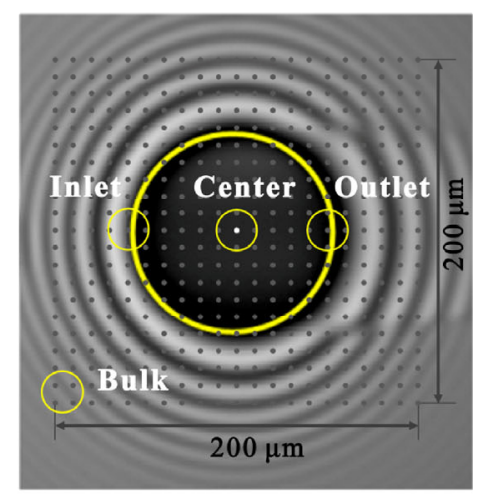

Fig. 1 (a) Schematic and (b) image of the experimental apparatus; (c) the schematic of the measuring points. 
than $5 \mathrm{~nm}$ according to the atomic-force microscopy measurements. Pure sliding conditions were maintained in all the experiments. When the glass disk started rotating, the lubricant molecules were entrained into the contact region, and a nanoscale lubricant film was formed. The applied load was $2.0 \mathrm{~N}$, and the diameter of the contact region was approximately $110 \mu \mathrm{m}$, with a maximum Hertz pressure of $312 \mathrm{MPa}$. The diameter of the steel ball is $12.7 \mathrm{~mm}$. In the distribution measurements, the linear sliding speed of the glass disk at the contact point was $31.4 \mathrm{~mm} / \mathrm{s}$ for Lubricants 1-4 and $12.6 \mathrm{~mm} / \mathrm{s}$ for Lubricants 5 and 6, to obtain TFL with a similar lubricant film thickness. Before each experiment, the ball and disk were carefully cleaned in an ultrasonic bath with petroleum ether and ethanol, respectively, for $20 \mathrm{~min}$.

In the Raman-measurement equipment, an Argon-ion laser with an emission wavelength of $514 \mathrm{~nm}$ was used as the Raman excitation source. The laser beam was focused on the lubricant film in the contact region through a long work distance objective with 50x magnification, as shown in Fig. 1(b). A quarter-wave plate was used to transform the linear polarized light emitted by the polarized laser into circularly polarized light. The scattered Raman radiation was collected by the objective, and Raman spectroscopy was finally acquired at $24{ }^{\circ} \mathrm{C}$ with a spectral resolution of $2 \mathrm{~cm}^{-1}$. The integral time for each spectrum was $30 \mathrm{~s}$, and the laser power was $30 \mathrm{~mW}$. The horizontal and vertical resolution of the Raman spectrum were both $2 \mu \mathrm{m}$. As the thickness of the lubricant film in the contact region was only in tens of nanometers, the Raman signal from the flat silica glass was collected by the spectrometer. The background signal was deducted and only signals from the lubricants were used for the data analysis. For a clear comparison, the exhibited Raman spectra in these measurements were normalized by dividing the spectrum by the intensity of $\mathrm{CH}_{3}$ at $2,890 \mathrm{~cm}^{-1}$.

The distribution profiles were mapped by collecting spectra at the points of a $200 \mu \mathrm{m} \times 200 \mu \mathrm{m}$ grid to evaluate the molecular distribution over the entire contact region, as shown in Fig. 1(c). The distance between every two points in the orthogonal direction was $10 \mu \mathrm{m}$. The diameter of the laser spot was $2 \mu \mathrm{m}$. The contact region is indicated by a large yellow circle. The inlet, outlet, and center of the contact region and the bulk region of the unconfined fluid are indicated by small yellow circles. To rapidly compare the distribution difference between the different lubricants, an average spectrum of four measuring points within the small circle is used as a reference. The Raman band of the characteristic vibration model of Lubricants 1-6 is given in Table 1.

Raman scattering occurs when a polarized laser beam illuminates the lubricant film, and the scattering strength depends on the change in photons energy [53]. According to Raman scattering theory [20], the scattering intensity of a certain molecule in the experiment system is given as follows:

$$
I_{i}=\frac{16 \pi^{4} \omega_{S i}^{4}}{c^{4}} A_{i} I_{0} N_{i}
$$

where $\omega_{\mathrm{S} i}$ is the frequency of the scattered light; $A_{i}$ is determined by the polarizability of the molecule; $I_{0}$ is the intensity of the incident light; and $N_{i}$ is the number of the molecules. In the Raman spectrum of a mixed lubricating liquid, the peak intensity ratio between components $\mathrm{a}$ and $\mathrm{b}$ is given as follows:

$$
\frac{I_{a}}{I_{b}}=\left(\frac{\omega_{\mathrm{S} a}}{\omega_{\mathrm{S} b}}\right)^{4} \frac{A_{a}}{A_{b}} \cdot \frac{N_{a}}{N_{b}}
$$

The Eq. (2) indicates that the intensity ratio is proportional to the volume ratio between two components. For the binary lubricants, the change in the characteristic peak intensity ratio $R_{\text {additive }}$ is used to evaluate the difference in the molecular distribution of the additive molecules in the contact region:

$$
R_{\text {additive }}=\frac{N_{\text {additive }}}{N_{\text {base off }}}=\frac{I_{\text {additive }}}{I_{\text {abse off }}}
$$

Where $I_{\text {additive }}$ is the Raman intensity of the characteristic peak of the additive molecule, while $I_{\text {abse off }}$ is that of the base oil. For Lubricant 2, 3, 4, and 6, the Eq. (3) can be applied directly to compare the molecular distribution of the additive at different positions of the contact region. On considering Lubricant 2 as an example, the benzene peak at $1,007 \mathrm{~cm}^{-1}$ is particular to phenethyl alcohol, and the $\mathrm{CH}_{3}$ symmetrical stretching vibration at $2,890 \mathrm{~cm}^{-1}$ is particular to hexadecane. Thus, the ratio of these two peaks can be used to evaluate the distribution of phenethyl alcohol 
in Lubricant 2. By mapping the Raman spectra at different positions of the contact region, the distribution profile of the additive in Lubricant 2 can be obtained. However, there is a slight difference for the situation of Lubricant 1 and 5 . For Lubricant 1 , the C-C stretching vibration at $1,607 \mathrm{~cm}^{-1}$ is particular to 5CB and the $\mathrm{CH}_{3}$ symmetrical stretching vibration at $2,890 \mathrm{~cm}^{-1}$ is common for $5 \mathrm{CB}$ and hexadecane. The intensity ratio between the peaks at $1,607 \mathrm{~cm}^{-1}$ and $2,890 \mathrm{~cm}^{-1}$ can be used to semi-quantitatively evaluate the distribution of 5CB in Lubricant 1.

\subsection{Molecular orientation measurements}

The molecular orientation and dynamics of the confined liquid thin film in the contact region were measured using the same in situ Raman-tribology system as that in the last section. However, the $1 / 4 \lambda$ plate in Fig. 1 was removed and an analyzer was placed in front of the entrance slit of the spectrometer. Only scattered light with a polarization that was parallel to the polarization direction of the incident laser was allowed to pass through the slit. The integral time for each spectrum was $30 \mathrm{~s}$, and the laser power was $30 \mathrm{~mW}$.

The $\mathrm{C}-\mathrm{C}$ aromatic vibration model of $5 \mathrm{CB}$ at $1,607 \mathrm{~cm}^{-1}$ was used to calculate the degree of orientational anisotropy, $S[53,54]$, which is defined as

$$
S=\frac{I_{\max (\theta)}-I_{\min (\theta)}}{I_{\max (\theta)}}
$$

where $\theta$ is the angle between the laser polarization and sliding direction, as shown in Fig. 2, and $I_{\max }$ (or $I_{\min }$ ) is the maximum (or minimum) Raman intensity in the $X Y$ plane. Theoretically, $S$ is zero when the lubricant molecules are macroscopically randomly

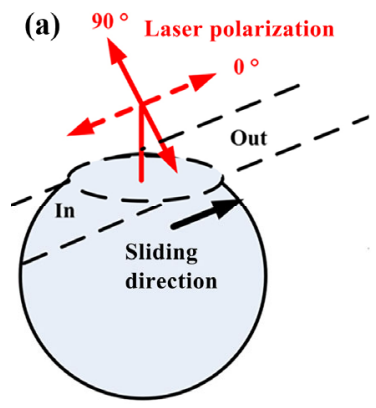

(b)

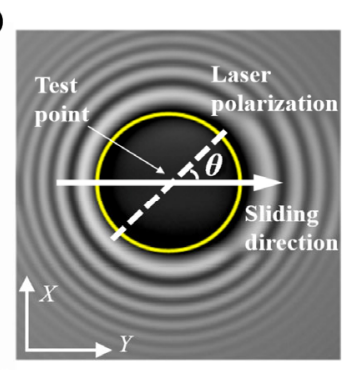

Fig. 2 (a) Schematic of the laser polarization and sliding direction; (b) the interference image of the contact region. oriented in the $X Y$ plane and 1 when all of the molecules are arranged in a uniform direction.

\subsection{Tribological measurements}

The viscosities of the six mixtures were measured using a rotational rheometer (MCR301, Co. Anton-Paar) at $24^{\circ} \mathrm{C}$, as shown in Table 1 . The standard viscosity measurements were performed in the rotational mode using a cone-plate system, and the angle between the surface of the cone and the plate is $2^{\circ}$ with a diameter of $50 \mathrm{~mm}$ (CP50-2/TP).

White light from a halogen lamp was used to observe the contact region using a microscope and a charge-coupled device camera. The central film thickness was measured from the interference fringes in an image obtained using the camera (the measurement of film thickness is described in detail in Refs. [22, 31, 55]). The resolution of the thickness measurement equipment was $0.5 \mathrm{~nm}$. The friction coefficient was measured using a ball-on-disk testing system (CETR-UMT-3, Bruker Optil $\mathrm{GmbH}$ ). The friction pair, load, velocity, temperature, as well as other conditions of the tribological experiments were the same as those in the in situ Raman measurements.

\section{Results and discussion}

\subsection{Effect of polarity on molecular distribution}

The distribution profile was successfully measured using our experimental apparatus and varied at different positions of the contact region. The distribution profile of Lubricant 1 in Fig. 3(b) shows that the intensity ratio increases rapidly as the lubricant runs into the contact region. In the center area, the intensity ratio reaches a maximum value of 10 , which is approximately 5.7 times that of the initial value in the bulk region. According to Eq. (3), the distribution ratio of 5CB in the lubricant film increases and is maximized at the center area. The 5CB molecules in Lubricant 1 exhibit an enrichment effect and are concentrated in the contact region during sliding. The intensity ratio gradually decreases to 1.7, thus approximating the value in the bulk region after the lubricant runs off. The enrichment effect disappears, and the molecular distribution returns to the initial value. The geometry of the confinement in the contact region or the interaction between 


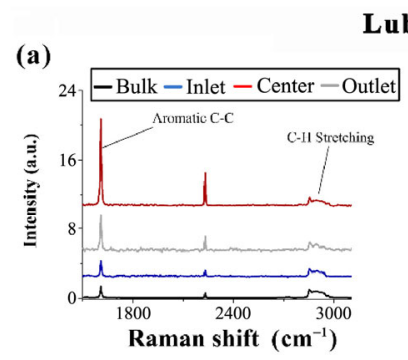

Lubricant 1

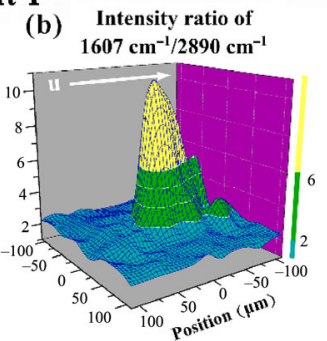

Lubricant 2

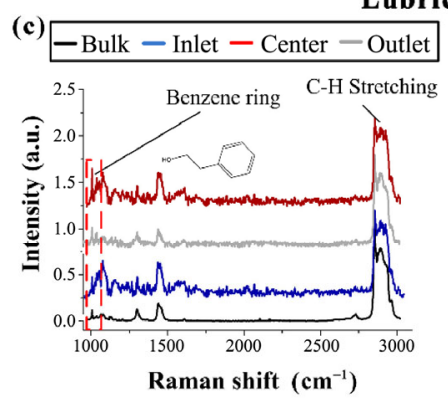

(d) Intensity ratio of $1007 \mathrm{~cm}^{-1} / 2890 \mathrm{~cm}^{-1}$

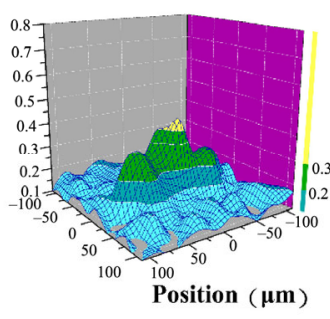

Lubricant 3

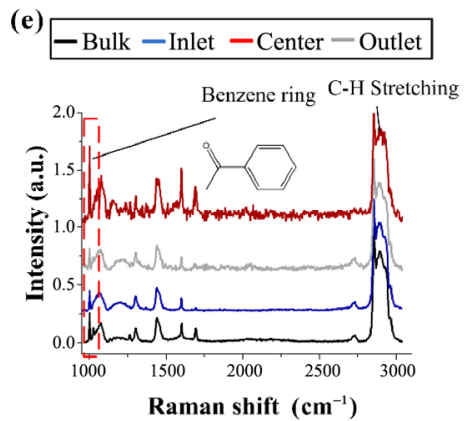

(f) Intensity ratio of $1002 \mathrm{~cm}^{-1} / 2890 \mathrm{~cm}^{-1}$

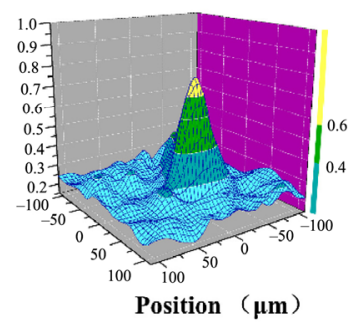

Lubricant 4

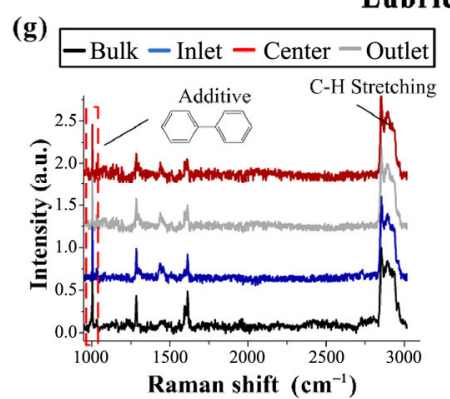

(h) Intensity ratio of $1004 \mathrm{~cm}^{-1} / 2890 \mathrm{~cm}^{-1}$

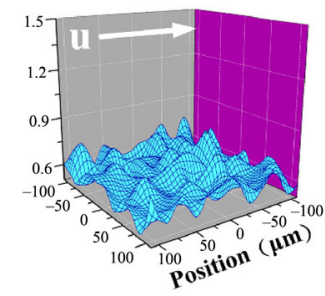

Fig. 3 Molecular distribution of Lubricant 1-4. (a), (c), (e), and (g): in situ Raman spectral results at the four areas and the molecular structures of two components; (b), (d), (e), and (h): in situ profile of the intensity ratio of the additive molecule to the base oil.

the lubricant and friction surface may be the reason for the enrichment effect, which is discussed below.

The polarities and functional groups of the additives have an influence on their molecular distribution. The additives in Lubricant $2-4$ are aromatic compounds with the same benzene ring and different terminal groups, including the alcohol group, ketone group, and benzene ring, respectively. All the base oils used are hexadecane. As shown in Figs.3(c)-3(h), polar phenethyl alcohol and acetophenone both exhibit an enrichment effect in hexadecane, while the nonpolar biphenyl does not. This indicates that the polar functional group of the additives, rather than the nonpolar benzene ring, is the main cause of the enrichment effect in the contact region. A new parameter $D_{\text {enrich }}$ (ratio of $R_{\text {additive }}$ at the center region and $R_{\text {additive }}$ at the bulk region) is raised to evaluate the degree of the enrichment effect. The $D_{\text {enrich }}$ values of phenethyl alcohol, acetophenone, and biphenyl are 2.92, 2.75, and 0 , respectively, which coincides with the strong-to-weak polarity order of their functional groups.

The polarity of the molecules is closely related to the interaction between the molecules and solid surface, and the surface anchoring of the heteroatoms in the polar functional group may result in an enrichment effect in the contact region. For Lubricant 1 , the $C \equiv N$ group of $5 \mathrm{CB}$ forms a bond with the steel and adopts a homeotropic arrangement, which is in accordance with some previous researches. The homeotropic arrangement was that the long axis of $5 \mathrm{CB}$ was parallel to the direction of the surface normal, which can be induced by a lecithin layer of hydrophobic surfactant monolayer [13]. Kumar et al. [6] found that $5 \mathrm{CB}$ aligned homeotropically on both the aminopropyl tri-ethoxy silane (APTES) and dimethyloctadecyl [3-(trimethoxysilyl) propyl] ammonium chloride (DMOAP) surfaces. Fourier transform infrared spectroscopy has confirmed that the $\mathrm{C} \equiv \mathrm{N}$ of $5 \mathrm{CB}$ interacted with $\mathrm{N}$ of APTES and $\mathrm{N}^{+}$of DMOAP $[6,17,56]$. Yang et al. [57] investigated the interfacial orientation of $5 \mathrm{CB}$ supported on salts of metal ions. It was found that metal ions with high electron affinities $(>15.64 \mathrm{eV}$, such as $\mathrm{Fe}^{3+}$ ) tended to induce a homeotropic orientation of $5 \mathrm{CB}$, because the nitrile group of $5 \mathrm{CB}$ coordinated with the metal ions. People also found that polar organic compounds with heteroatoms such as nitrogen and oxygen easily formed bond between the heteroatom electron cloud and metal and adsorbed onto the steel surface, thus acting as a corrosion inhibitor [9-11]. In our research, polar 5CB molecules were adsorbed onto the steel surface and adopted a homeotropic arrangement, while nonpolar hexadecane did not adsorb onto the surface. 
The base oils in Lubricant 1-4 are all hexadecane, which is often considered as a nonpolar molecule. The additives, 5CB, phenethyl alcohol, and acetophenone in Lubricant 1-3 are all polar molecules. The three polar molecules are all concentrated in the contact region. Their polar terminal groups with the nitrogen and oxygen heteroatom make it easier to adsorb onto a metal surface and form bonds between the heteroatom electron cloud and the metal as compared with the nonpolar hexadecane without a heteroatom. Polar additive molecules stay in the contact region while the majority of the nonpolar base oil molecules run off with the flow of the lubricants. Thus, the polar additive molecules are concentrated in the nanogap, and the intensity ratio in the contact region is higher than that in the bulk region.

The polarity and functional groups of the base oil also have a significant influence on the distribution of the additive molecules. The base oils in Lubricant 1, 5, and 6 are hexadecane, decyl alcohol, and phenethyl alcohol. The additives are all 5CB. As shown in Figs. 3(b), 4(b) and 4(d), 5CB molecules, as additives, exhibit a strong enrichment effect in the nonpolar hexadecane base oil, but no enrichment in polar base oils such as decyl alcohol and phenethyl alcohol. The two polar base oils both have hydroxyl groups. The additive and base oil molecules in Lubricant 5 and 6 both tend to adsorb onto the metal surface and compete with each other. An equilibrium state is reached, and the intensity ratio $R_{\text {additive }}$ in the contact region is nearly the same as that in the bulk fluid.

\subsection{Tribological performance}

The friction coefficient and lubricant film thickness, which are important parameters of tribological performance, are closely related to the molecular distribution of the additives. The friction coefficient of Lubricant 1-4 was measured under the same conditions as the Raman experiment, as shown in Fig. 5. The friction coefficient of pure hexadecane was also measured as a reference. The results show that the hexadecane lubricants with $5 \mathrm{CB}$, phenethyl alcohol, and acetophenone additives reduce the friction coefficient to some extent. In particular, 5CB additives exhibit the best performance with $61 \%$ reduction of friction coefficient, while biphenyl shows no friction reduction
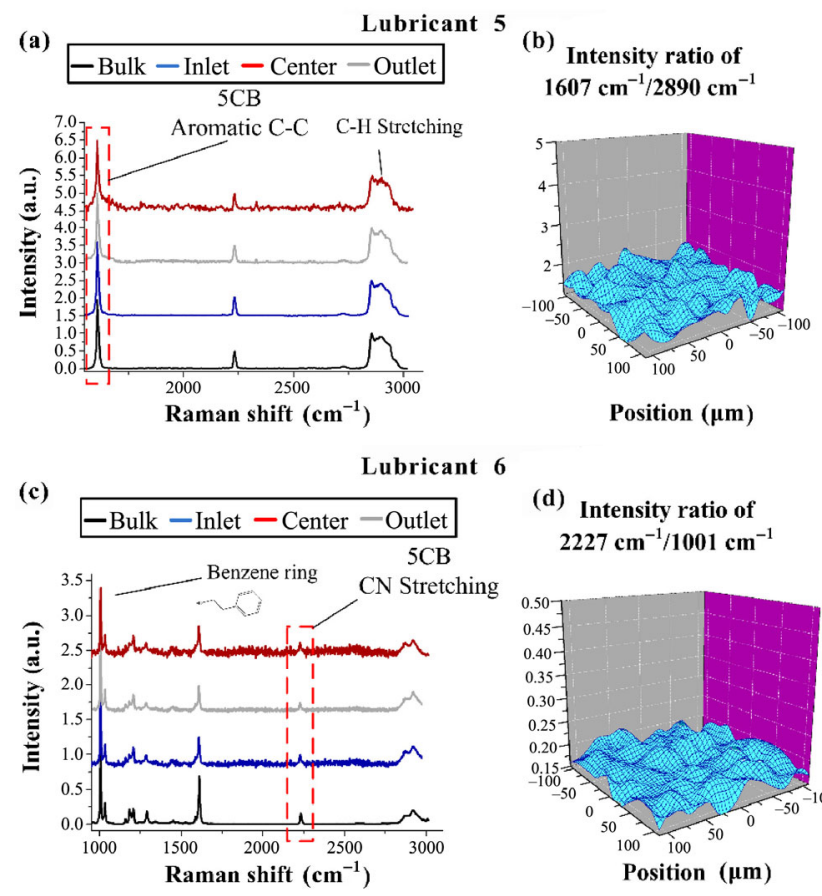
(d) Intensity ratio of
$2227 \mathrm{~cm}^{-1} / 1001 \mathrm{~cm}^{-1}$

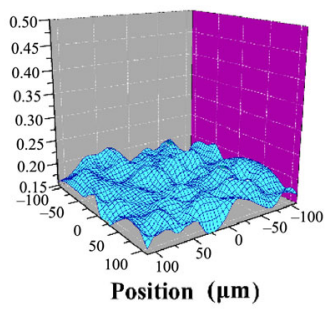

Fig. 4 Molecular distribution of Lubricant 5 and 6. (a) and (c): in situ Raman spectral results at the four areas and the molecular structures of two components; (b) and (d): in situ profile of the intensity ratio of additive molecule to base oil.

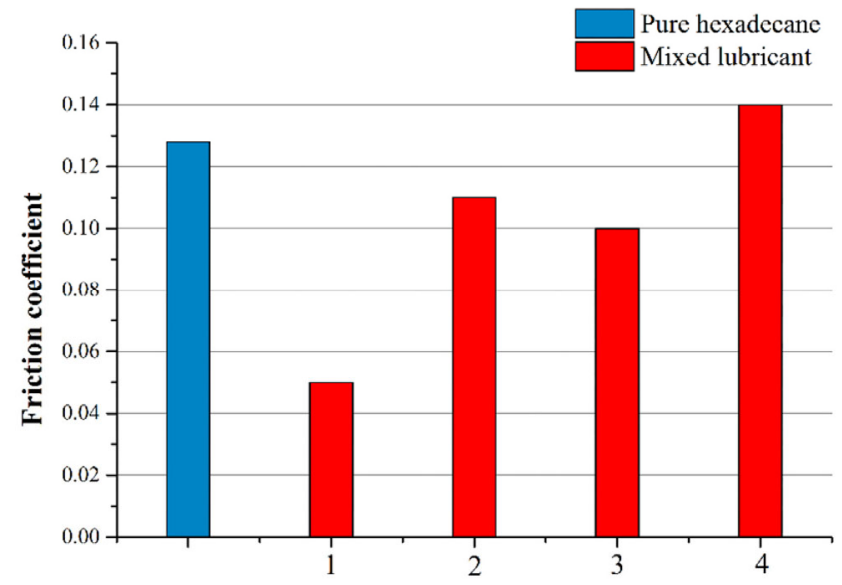

Fig. 5 Friction coefficient of pure hexadecane and Lubricant $1-4$.

effect. The friction coefficient decreases as the concentration of the additive increases. This result is in accordance with the experiments on $5 \mathrm{CB}$ with the hexadecane base oil that were conducted by Yao et al. [58]. They found that the $5 \mathrm{CB}$ additive significantly reduced the friction and wear, and this friction-reducing ability improved when the concentration of $5 \mathrm{CB}$ increased in the range of $1.0 \mathrm{wt} . \%$ to $5.0 \mathrm{wt} . \%$. It was believed that the adsorption of $5 \mathrm{CB}$ on a steel surface 
played an important role in the reduction of friction, as the existence of 5CB molecules on the steel surface was confirmed by micro-reflection infrared spectroscopy. Itoh et al. [19] also proposed that the slip of the 5CB layer adjacent to the solid surface resulted in low friction and a high load capacity. In our research, the friction-reduction ability of the additive is related to the molecular distribution and may result from the thin adsorption layer near the solid surface. The adsorption layer prevents the direct contact of asperities and helps reduce the friction coefficient.

Additives in the lubricant are concentrated in the contact region and form a thin adsorption layer. The thickness of the lubricant film should increase with the strength of the enrichment effect. It is supported by the measured center film thickness of Lubricant 1-4. As shown in Fig. 6, the intensity ratio in the bulk region is normalized to $100 \%$, and the ratio between the center and bulk regions represents the strength of the enrichment effect. $5 \mathrm{CB}$ exhibits the strongest enrichment effect and the thickest film thickness of $16.0 \mathrm{~nm}$. The enrichment strength and the film

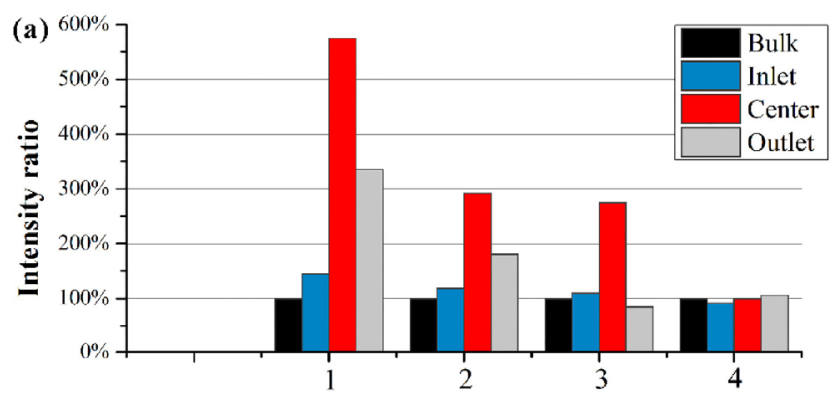

(b)

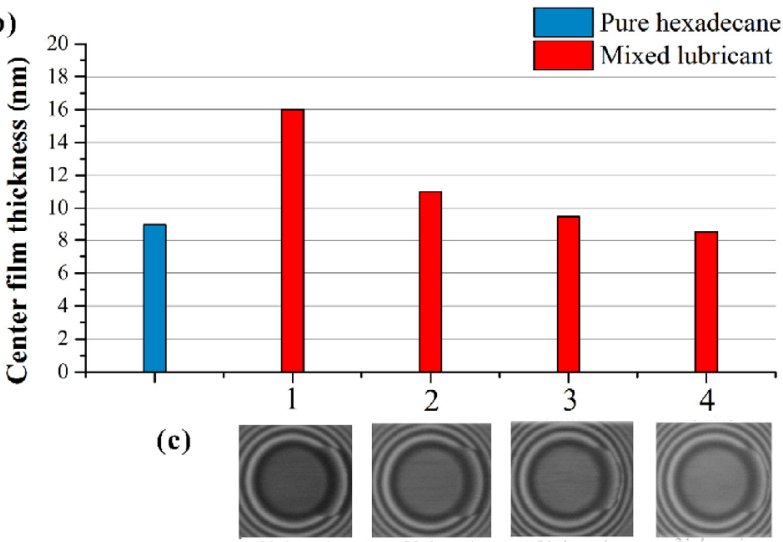

Fig. 6 (a) Relative change in intensity ratio in different areas of the contact region as compared with that in the bulk region $(100 \%)$; (b) the center film thickness; (c) the interference image in the contact region of Lubricant 1-4. thickness of phenethyl alcohol and acetophenone are at a same level. Biphenyl exhibits no enrichment effect, and its film thickness is close to the value of pure hexadecane.

\subsection{Binary multilayer model}

The film thicknesses of Lubricant 1-4 between the glass disk and steel ball are less than $20 \mathrm{~nm}$, which is in the range of TFL. The use of a binary multilayer model is proposed to describe the molecular behavior in TFL. Lubricant 1-3 are presented in Fig. 7(a). The polar additive molecules adsorb onto the steel surface, thus forming an adsorption layer with a homeotropic alignment. The volume of the additive molecules in the adsorption layer is much greater than that of the base oil. The distribution ratio of the additive in the fluid layer is close to the liquid outside the contact region, as the fluid layer is less affected by surface adsorption. The fluid layer in the TFL is nearly negligible, and the molecular distribution in the contact region is dominated by the adsorption layer. Although surface adsorption dominates in the adsorption layer, weak shear induction still exists near the surface and results in a very small angle between the molecule director and surface normal direction. Lubricant 4 is presented in Fig. 7(c). The ordered adsorption layer is not formed in the absence of polar molecules. The distribution ratio of the additive in the lubricant film is close to the bulk liquid outside the contact region and exhibits no enrichment effect. Lubricant 5 and 6 are presented in Fig. 7(b). The adsorption layer is formed by the competition of the polar additive and polar base oil. Without priority, the distribution ratio of the additive in the lubricant is also similar to the bulk fluid and exhibits no enrichment effect. The molecules in the adsorption layer also adopt an

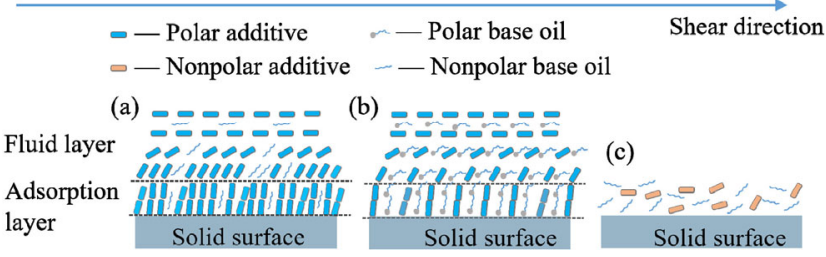

Fig. 7 Schematic of the binary multilayer model in TFL in the contact region: (a) polar additive in nonpolar base oil; (b) polar additive in polar base oil; and (c) nonpolar additive in nonpolar base oil. 
approximately perpendicular arrangement under the surface adsorption, steric hindrance, and shear.

The model is verified by changing the thickness of the fluid layer. For Lubricant $1-4$, the sliding speed is increased to $188.4 \mathrm{~mm} / \mathrm{s}$, which is in the EHL regime. As shown in Figs. 6(b) and 8, the measured film thickness under a sliding speed of $188.4 \mathrm{~mm} / \mathrm{s}$ is greater than that under $31.4 \mathrm{~mm} / \mathrm{s}$. The intensities of the characteristic Raman peaks of the additive and base oil in the center region and bulk liquid are almost the same. The intensity ratio of the characteristic Raman bands remains constant, which indicates no enrichment effect. The enrichment effect of the additive disappears with the increase in the lubricant film thickness. The thickness of the adsorption layer keeps constant, while that of the fluid layer gets much thicker. Thus, the distribution of the additive molecules is dominated by the fluid layer.

\subsection{Effect of polarity on molecular orientation}

Our previous research [20] confirmed that the friction coefficient of pure $5 \mathrm{CB}$ decreased as the orientational anisotropy of the $5 \mathrm{CB}$ molecule increased. The interfacial orientation of the molecules has attracted attention not only in tribology but also in surface science. Many studies have been conducted, and it is
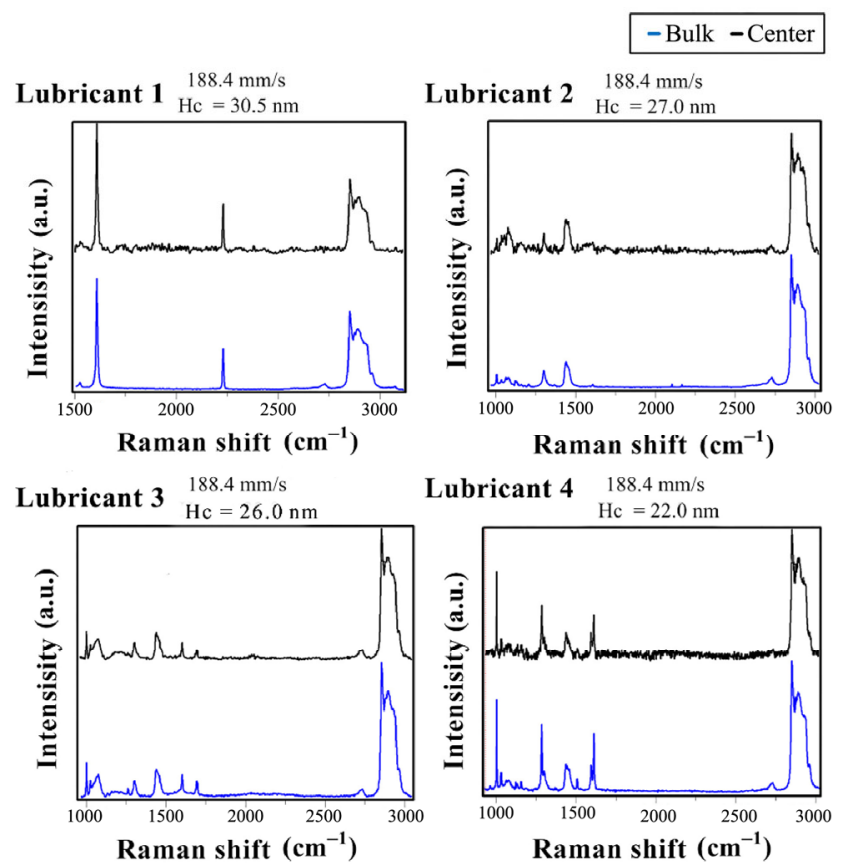

Lubricant $4 \begin{gathered}188.4 \mathrm{~mm} / \mathrm{s} \\ \mathrm{Hc}=22.0 \mathrm{~nm}\end{gathered}$

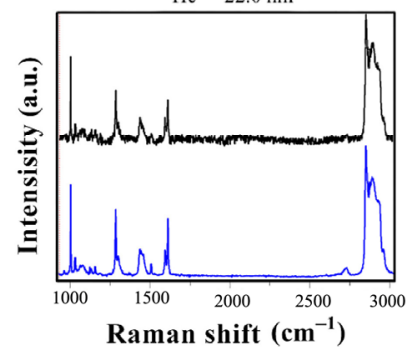

Fig. 8 In situ Raman spectral results for Lubricant 1-4 at different areas and the measured film thickness in the center region. confirmed that the shear alignment, confinement, and surface adsorption can exist independently and influence the molecular orientation. It is well known that a nematic liquid crystal such as $5 \mathrm{CB}$ can retain a long-range orientational order under shear [12, 17]. When the shear rate is higher than the rate of molecular rearrangement, slip occurs between the molecules, and the viscosity decreases with the increasing shear rate. This is called shear thinning and may be the reason that $5 \mathrm{CB}$ molecules can cause a reduction in friction when used as a lubricant or lubricant additive [19]. Confinement plays an important role in molecular alignment. In our previous research [20], the orientational anisotropy of $5 \mathrm{CB}$ in the contact region is even greater than that outside the contact region. This indicates that confinement in the nanometer gap promotes the alignment of 5CB. Confinement improves the positional order and induces a liquid-to-solid phase transition. Furthermore, the induction of confinement can exist independent of shear [13, 59]. Liquid crystal molecules can adopt both planar and homeotropic alignment on different treated substrates. Planar alignment is usually obtained by a method of mechanical rubbing or photoalignment [17]. Homeotropic alignment is favored on hydrophobic surfaces, which can be obtained by adsorbing a lecithin or surfactant monolayer. On the metal surface, 5CB molecules exhibit a homeotropic alignment because of the coordination between $\mathrm{C} \equiv \mathrm{N}$ and metal ions. In the adsorption layer of our binary multilayer model, polar 5CB molecules are adsorbed on the steel ball surface and adopt a homeotropic arrangement.

However, there is lack of comprehensive research on the orientation of liquid crystals in a multiphase liquid. Das et al. [60] mixed 5CB and mesogen (ME50.5) and studied the molecular organization and newly formed smectic phase. Kato et al. [61] mixed 5CB with an anthracene derivative and studied the formation of organic physical gels. In the field of tribology, the orientation of the $5 \mathrm{CB}$ additive in base oil and the influence of the polarity of the base oil are explored in this section.

The molecular orientation of the $5 \mathrm{CB}$ additive was evaluated using orientational anisotropy $S$, which was measured under a series of sliding speeds. The anisotropic structure of the $5 \mathrm{CB}$ molecule results in a directional response to laser polarization. In the 
experiment, when the molecular axis of $5 \mathrm{CB}$ is parallel to the laser polarization, the intensity of the characteristic peak reaches a maximum value.

Shear in the sliding process induces the liquid crystal molecules in the binary mixture to become oriented along the flow direction. However, the degree of this orientation is lower than that of pure liquid crystals. According to our previous study, under the same sliding speed of $31.4 \mathrm{~mm} / \mathrm{s}$, pure $5 \mathrm{CB}$ exhibited an orderly arrangement with $S=0.58$, and pure hexadecane exhibited a nearly isotropic orientation with $S=0.2$ [20]. Shear stress and confinement promote a parallel molecular alignment along the shear direction but surface adsorption induces a homeotropic arrangement of $5 \mathrm{CB}$ on a steel surface. A combination of these three factors results in an intermediate strength of orientational anisotropy of 0.58 for 5CB. When mixing $5 \mathrm{CB}$ and hexadecane together, as shown in Figs. 9(a) and 9(b), the polarization intensity of $5 \mathrm{CB}$ along the sliding direction is greater than that in the other directions, with a calculated $S$ of 0.27 . This demonstrates that the majority of the $5 \mathrm{CB}$ molecules in hexadecane tend to be oriented along the sliding direction because of the shear inducement. As compared with the pure state, $5 \mathrm{CB}$ molecules exhibit a lower degree of orientational anisotropy under the hindrance of disordered hexadecane.
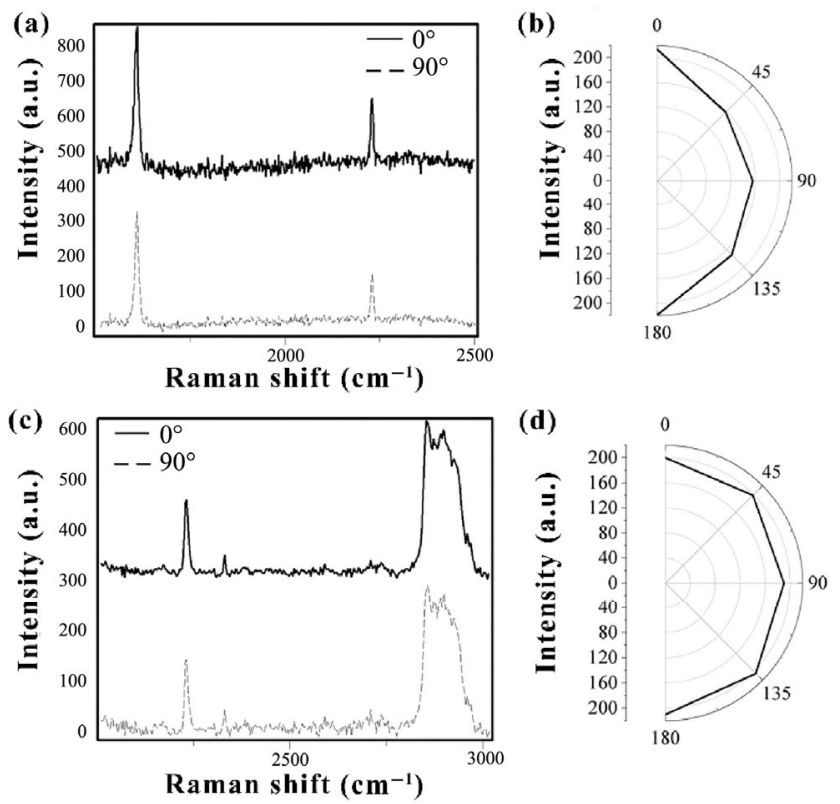

Fig. 9 In situ Raman spectral results of Lubricant 1-4 at different areas and the measured film thickness at the center region.
The effect of polarity on the molecular orientation was investigated by changing the base oil from nonpolar hexadecane (Lubricant 1) to polar decyl alcohol (Lubricant 5). The molecular structure of decyl alcohol is almost the same as that of hexadecane except for a terminal hydroxy. As shown in Figs. 9(c) and $9(\mathrm{~d})$, the polarization intensities along the different angles remain approximately unchanged, and the calculated $S$ value is 0.07 . This indicates that $5 \mathrm{CB}$ molecules in decyl alcohol adopt an isotropic arrangement. The polar terminal hydroxy group has an obvious effect on the orientation of $5 \mathrm{CB}$ liquid molecules. Two factors may significantly affect the molecular arrangement. Firstly, the enrichment effect of $5 \mathrm{CB}$ disappears as the polar hydroxy competes with $5 \mathrm{CB}$ for adsorption on the solid surface. The lack of a condensed adsorption layer results in a weak intermolecular interaction of $5 \mathrm{CB}$ as compared with that in Lubricant 1 . The long-rang order of $5 \mathrm{CB}$ in Lubricant 5 is interrupted, and the orientational anisotropy is weaker. Secondly, polar decyl alcohol is a type of chiral molecule [62], which may induce $5 \mathrm{CB}$ to form a helical structure. It is found that chiral dopants generate strong helicity in the nematic phase, and the strength of a dopant to generate a helical structure is called helical twisting power (HTP). Rudquist et al. [63] found that 5CB molecules formed a helical structure when doped with chiral mandelic acid with hydroxy. Kishikawa et al. [64] found that the substituents of a series of chiral succinimide derivative significantly affected the formation of a helical structure of $5 \mathrm{CB}$, and the derivative with a terminal hydroxyl resulted in a larger HTP than the derivative with a terminal alkyl. Thus, doping decyl alcohol may result in a helical and isotropic structure of 5 CB.

The geometry of confinement, i.e., the thickness of the lubricant specifically, has an influence not only on the molecular distribution of the additive, but also on the molecular orientation. The thickness of the lubricating film increases and the lubricating state changes as the sliding speed increases. The lubricating state is TFL when the speed is less than $31.4 \mathrm{~mm} / \mathrm{s}$. It is EHL when the speed is greater than $62.8 \mathrm{~mm} / \mathrm{s}$. The range of speeds between $31.4 \mathrm{~mm} / \mathrm{s}$ and $62.8 \mathrm{~mm} / \mathrm{s}$ is a transition state from TFL to EHL. The variation in $S$ of pure $5 \mathrm{CB}$ and $5 \mathrm{CB}$ in hexadecane are shown in 
Fig. 10(a). In TFL, with a sliding speed of $12.6 \mathrm{~mm} / \mathrm{s}$, two lubricants exhibit a similar $S$ of nearly 0.2 , which indicates a weak orientation along the sliding direction. In the adsorption layer, 5CB molecules adopt an approximately perpendicular arrangement that is slightly along the sliding direction, under the dominance of surface adsorption and the assistance of shear. The adsorption layer dominates in TFL, and 5CB molecules exhibit a weak orientation along the flow, as the lubricating film thickness is approximately $15 \mathrm{~nm}$. The lubricating film thicknesses of pure $5 \mathrm{CB}$ and $5 \mathrm{CB}$ in hexadecane increase with the sliding speed, as shown in Fig. 10(b). 5CB molecules become oriented along the sliding direction because of the dominance of shear induction. The fluid layer gradually dominates when it transitions into EHL. Thus, $S$ increases with the sliding speed and reaches a constant value in EHL. The confinement and shear effect on molecular orientation is the same because of the same centerfilm thickness and sliding velocity. The only reason for a lower $S$ of $5 \mathrm{CB}$ in hexadecane than pure $5 \mathrm{CB}$ is that the disordered hexadecane restrains the orderly alignment of 5CB. The $S$ value of 5CB in decyl alcohol is independent of the sliding speed and remains at a low value of nearly 0.2 , thus indicating that the isotropy orientation of $5 \mathrm{CB}$ is dominated by the intermolecular interaction between 5CB and decyl alcohol, instead of confinement and shear induction.

The behaviors of molecules in the bulk fluid layer have been systemically investigated. However, the layered structure in TFL is still calling for direct observation and study, especially molecules in the absorbed layer and ordered layer. In the work presented in ref. [65], we have successfully detected and shown both the packing and orienting of liquid molecules in the absorbed layer and ordered layer in TFL regime.
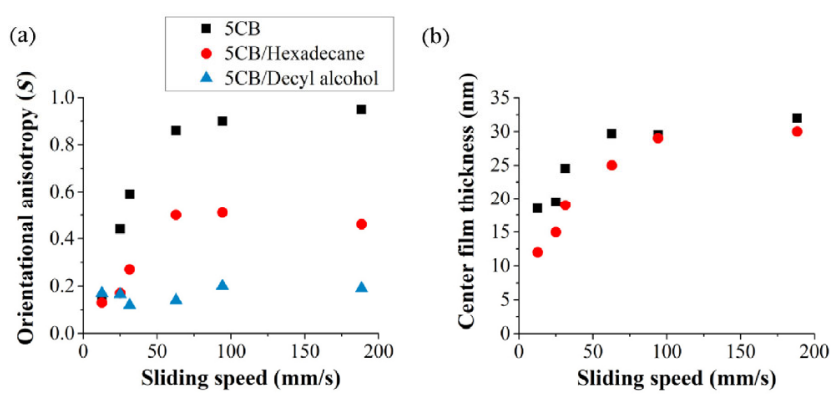

Fig. 10 (a) Variation of $S$ with sliding speed; (b) the variation of lubricating film thickness with sliding speed.

\section{Conclusions}

The molecular distribution profile and orientation of additives in binary mixed lubricants were investigated at the nanoscale. In situ Raman experiments were conducted under pure sliding conditions. The results showed that polar additives concentrated in the contact region when added into a nonpolar base oil, but the concentration phenomenon disappeared with a polar base oil. This was attributed to the competition of surface anchoring between the polar additives and base oils. By using liquid crystal molecules and a nonpolar base oil as lubricant, the friction coefficient was significantly reduced by $61 \%$. The lubricating ability was related to the adsorption layer and the ordered arrangement of the liquid crystal molecules, which was verified via polarized Ramen experiments. Shear stress induced a molecular alignment along the shear direction, and confinement in the nanogap promoted a more ordered alignment. Surface adsorption affected the lubricant in a different manner and induced a perpendicular arrangement of $5 \mathrm{CB}$ on the steel surface. A combination of these three factors determined the molecular orientation in the tribology system. The effect of the polarities of molecules in binary lubricants on the molecular orientation was investigated. It was found that the surface adsorption was dominated by the polarities of molecules, and the molecular arrangement was affected by a combination of surface adsorption, confinement, and shear stress. The lubrication mechanism model of the binary lubricants was developed to describe the molecular behavior in TFL. Our research may aid in understanding nanoscale lubricating mechanisms and the development of novel liquid lubricants.

\section{Acknowledgements}

The work was financially supported by the National Natural Science Foundation of China (No. 51875303 and No. 51527901).

Open Access This article is licensed under a Creative Commons Attribution 4.0 International License, which permits use, sharing, adaptation, distribution and reproduction in any medium or format, as long as you 
give appropriate credit to the original author(s) and the source, provide a link to the Creative Commons licence, and indicate if changes were made.

The images or other third party material in this article are included in the article's Creative Commons licence, unless indicated otherwise in a credit line to the material. If material is not included in the article's Creative Commons licence and your intended use is not permitted by statutory regulation or exceeds the permitted use, you will need to obtain permission directly from the copyright holder.

To view a copy of this licence, visit http://creativecommons.org/licenses/by/4.0/.

\section{References}

[1] Pizzirusso A, Berardi R, Muccioli L, Ricci M, Zannoni C. Predicting surface anchoring: Molecular organization across a thin film of $5 \mathrm{CB}$ liquid crystal on silicon.ChemSci 3(2): 573-579 (2012)

[2] Yang Y M, Chen W, Dou L T, Chang W H, Duan H S, Bob B, Li G, Yang Y. High-performance multiple-donor bulk hetero junction solar cells. Nat Photonics 9(3): 190-198 (2015)

[3] Chen W, Xu T, He F, Wang W, Wang C, Strzalka J, Liu Y, Wen J G, Miller D J, Chen J H, et al. Hierarchical nanomorphologies promote exciton dissociation in polymer/ fullerene bulk hetero junction solar cells. Nano Lett 11(9): 3707-3713 (2011)

[4] Dave S R, Gao X H. Monodisperse magnetic nanoparticles for biodetection, imaging, and drug delivery: A versatile and evolving technology. Wiley Interdiscip Rev-Nanomed Nanobiotechnol 1(6): 583-609 (2009)

[5] Toma A C, Dootz R, Pfohl T. Analysis of complex fluids using microfluidics: The particular case of DNA/polycations assemblies. J Phys D: Appl Physi 46(11): 114001 (2013)

[6] Kumar A, Pattanayek S K. Exploitation of orientation of liquid crystals $5 \mathrm{CB}$ and DSCG near surfaces to detect low protein concentration. Liquid Crystals 42(10): 1506-1514 (2015)

[7] Li X W, Reinhoudt D, Crego-Calama M. What do we need for a superhydrophobic surface? A review on the recent progress in the preparation of superhydrophobic surfaces. Chem Soc Rev 36(8): 1350-1368 (2007)

[8] Feng L, Song Y L, Zhai J, Liu B Q, Xu J, Jiang L, Zhu D B. Creation of a superhydrophobic surface from an amphiphilic polymer. Angew Chem Int Ed 42(7): 800-802 (2003)

[9] Kalin M, Vižintin J, Vercammen K, Barriga J, Arnšek A. The lubrication of DLC coatings with mineral and biodegradable oils having different polar and saturation characteristics. Surf Coat Techno 200(14-15): 4515-4522 (2006)
[10] Li W H, He Q, Pei C L, Hou B R. Experimental and theoretical investigation of the adsorption behaviour of new triazole derivatives as inhibitors for mild steel corrosion in acid media. Electrochim Acta 52(22): 6386-6394 (2007)

[11] Hegazy M A, Hasan A M, Emara M M, Bakr M F, Youssef A H. Evaluating four synthesized Schiff bases as corrosion inhibitors on the carbon steel in $1 \mathrm{M}$ hydrochloric acid. Corros Sci 65: 67-76 (2012)

[12] Silva B F B, Zepeda-Rosales M, Venkateswaran N, Fletcher B J, Carter L G, Matsui T, Weiss T M, Han J, Li Y L, Olsson $\mathrm{U}$, et al. Nematic director reorientation at solid and liquid interfaces underflow: SAXS studies in a microfluidic device. Langmuir 31(14): 4361-4371 (2015)

[13] Ruths M, Steinberg S, Israelachvili J N. Effects of confinement and shear on the properties of thin films of thermotropic liquid crystal. Langmuir 12(26): 6637-6650 (1996)

[14] Cramer C, Cramer T, Kremer F, Stannarius R. Measurement of orientational order and mobility of a nematic liquid crystal in random nanometer confinement. $J$ Chem Phys 106(9): 3730-3742 (1997)

[15] Iannacchione G, Finotello D. Confinement and orientational study at liquid crystal phase transitions. Liq Cryst 14(4): 1135-1142 (1993)

[16] Iannacchione G S, Crawford G P, Doane J W, et al. Orientational effects on confined 5CB. Mol Cryst Liq Cryst 222: 205-213 (1992)

[17] Sengupta A, Schulz B, Ouskova E, Bahr C. Functionalization of microfluidic devices for investigation of liquid crystal flows. Microfluid Nanofluid 13(6): 941-955 (2012)

[18] Gao Y, Xue B C, Ma L R, Luo J B. Effect of liquid crystal molecular orientation controlled by an electric field on friction. Tribol Int 115: 477-482 (2017)

[19] Itoh S, Imura Y, Fukuzawa K, Zhang H D. Anisotropic shear viscosity of photo aligned liquid crystal confined in submicrometer-to-nanometer-scale gap widths revealed with simultaneously measured molecular orientation. Langmuir 31(41): 11360-11369 (2015)

[20] Zhang S H, Liu Y H, Luo J B. In situ observation of the molecular ordering in the lubricating point contact area. $J$ Appl Phys 116(1): 014302 (2014)

[21] Luo J B. Study on the measurement and experiments of thin film lubrication. Ph.D. Thesis. Beijing (China): Tsinghua University, 1994.

[22] Luo J B, Wen S Z, Huang P. Thin film lubrication. Part I. Study on the transition between EHL and thin film lubrication using a relative optical interference intensity technique. Wear 194(1-2): 107-115 (1996)

[23] Luo J B, Wen S Z. Mechanism and characters of thin film lubrication at nanometer scale. Sci China SerA-Math 39(12): 1312-1322 (1996) 
[24] Luo J B, Wen S Z, Sheng X Y, Li L K Y. Substrate surface energy effects on a liquid lubricant film at nanometer scale substrate surface energy effects on a liquid lubricant film at nanometre scale. Lubr Sci 11(1): 23-36 (1998)

[25] Ma L R, Luo J B. Thin film lubrication in the past 20 years. Friction 4(4): 280-302 (2016)

[26] Spikes H A, Guangteng G. Paper XI(i) properties of ultrathin lubricating films using wedged spacer layer optical interferometry. Tribology 12: 275-279 (1987)

[27] Lee S, Müller M, Ratoi-Salagean M, VörösJ, Pasche S, De Paul S M, Spikes H A, Textor M, Spencer N D. Boundary lubrication of oxide surfaces by poly(L-lysine)- $g$ - poly(ethylene glycol) (PLL-g-PEG) in aqueous media. Tribol Lett 15(3): 231-239 (2003)

[28] Ratoi M, Spikes H A. Lubricating properties of aqueous surfactant solutions. Tribol Trans 42(3): 479-486 (1999)

[29] Taylor L J, Spikes H A. Friction-enhancing properties of ZDDP antiwear additive: Part I-friction and morphology of ZDDP reaction films. Tribol Trans 46(3): 303-309 (2003)

[30] Matsuoka H, Kato T. An ultrathin liquid film lubrication theory-calculation method of solvation pressure and its application to the EHL problem. J Tribol 119(1): 217-226 (1997)

[31] Ma L R, Zhang C H. Discussion on the technique of relative optical interference intensity for the measurement of lubricant film thickness. Tribol Lett 36(3): 239-245 (2009).

[32] Luo J B. Thin film lubrication and lubrication map. (in Chinese). Chin J Mech Eng 36(36): 5-10 (2000)

[33] Luo J B, Shen M W, Wen S Z. Tribological properties of nanoliquid film under an external electric field. $J$ Appl Phys 96(11): 6733-6738 (2004)

[34] Xie G X, Luo J B, Liu S H, Guo D, Zhang C H. "Freezing" of nanoconfined fluids under an electric field. Langmuir 26(3): 1445-1448 (2010)

[35] Xie G X, Luo J B, Guo D, Liu S H. Nanoconfined ionic liquids under electric fields. Appl Phys Lett 96(4): 043112 (2010)

[36] Hartl M, Krupka I, Liska M. Differential colorimetry: Tool for evaluation of chromatic interference pattern. Opt Eng 36(9): 2384-2391 (1997)

[37] Křupka I, Hartl M, Liška M. Thin lubricating films behaviour at very high contact pressure. Tribol Int 39(12): 1726-1731 (2006)

[38] Sperka P, Krupka I, Hartl M. Lubricant flow in thin-film elastohydrodynamic contact under extreme conditions. Friction 4(4): 380-390 (2016)

[39] Guo L, Wong P, Guo F. Identifying the optimal interfacial parameter correlated with hydrodynamic lubrication. Friction 4(4): 347-358 (2016)
[40] Zhang Y G, Wang W Z, Zhang S G, Zhao Z Q. Optical analysis of ball-on-ring mode test rig for oil film thickness measurement. Friction 4(4): 324-334 (2016)

[41] Cann P M. In-contact molecular spectroscopy of liquid lubricant films. MRS Bull 33(12): 1151-1158 (2008)

[42] Muratore C, Bultman J E, Aouadi S M, Voevodin A A. In situ Raman spectroscopy for examination of high temperature tribological processes. Wear 270(3-4): 140-145 (2011)

[43] Bongaerts J H H, Day J P R, Marriott C, Pudney P D A, Williamson A M. In situ confocal Raman spectroscopy of lubricants in a soft elastohydrodynamic tribological contact. J Appl Phys 104(1): 014913 (2008)

[44] Beattie D A, Winget S A, Bain C D. Raman scattering from confined liquid films in the sub-nanometre regime. Tribol Lett 27(2): 159-167 (2007)

[45] Beattie D A, Haydock S, Bain C D. A comparative study of confined organic monolayers by Raman scattering and sumfrequency spectroscopy. Vib Spectrosc 24(1): 109-123 (2000)

[46] Bae S C, Lee H, Lin Z Q, Granick S. Chemical imaging in a surface forces apparatus: Confocal Raman spectroscopy of confined poly(dimethylsiloxane). Langmuir 21(13): 5685-5688 (2005)

[47] Nanjundiah K, Hsu P Y, Dhinojwala A. Understanding rubber friction in the presence of water using sum-frequency generation spectroscopy. J Chem Phys 130(2): 024702 (2009)

[48] Golan Y, Martin-Herranz A, Li Y L, Safinya C R, Israelachvili J. Direct observation of shear-induced orientational phase coexistence in a lyotropic system using a modified x-ray surface forces apparatus. Phys Rev Lett 86(7): 1263-1266 (2001)

[49] Hsu S, Ying C, Zhao F. The nature of friction: A critical assessment. Friction 2(1): 1-26 (2014)

[50] Bae S C, Wong J S, Kim M, Jiang S, Hong L, Granick S. Using light to study boundary lubrication: Spectroscopic study of confined fluids. Philos Trans R Soc A: Math, Phys Eng Sci 366(1869): 1443-1454 (2008)

[51] Yi J, Jonas J. Raman study of vibrational and rotational relaxation of liquid benzene- $d_{6}$ confined to nanoporous silica glasses. J Phys Chem 100(42): 16789-16793 (1996)

[52] Snyder R G, Strauss H L, Elliger C A. Carbon-hydrogen stretching modes and the structure of n-alkyl chains. 1. Long, disordered chains. J Phys Chem 86(26): 5145-5150 (1982)

[53] Raman C V, Krishnan K S. A new type of secondary radiation. Nature 121(3048): 501-502 (1928)

[54] Gao M, Ma L R, Luo J B. Effect of alkyl chain length on the orientational behavior of liquid crystals Nano-film. Tribol Lett 62(2): 24 (2016)

[55] Liang H, Guo D, Luo J. Film forming behavior in thin film lubrication at high speeds. Friction 6(2): 156-163 (2018) 
[56] Jerome B. Surface effects and anchoring in liquid crystals. Rep Prog Phys 54(3): 391-451 (1991)

[57] Yang K L, Cadwell K, Abbott N L. Mechanistic study of the anchoring behavior of liquid crystals supported on metal salts and their orientational responses to dimethyl methylphosphonate. J Phys Chem B 108(52): 20180-20186 (2004)

[58] Yao J B, Wang G L, Xu Z, Wen S Z. The tribological performance of nematic liquid crystal 4-n-pentyl-4'-cyanobiphenyl (5CB) as a lubricant additive. Lubr Eng 56(6): 21-25 (2000)

[59] Janik J, Tadmor R, Klein J. Shear of molecularly confined liquid crystals. 1 . Orientation and transitions under confinement. Langmuir 13(16): 4466-4473 (1997)

[60] Das M K, Paul R, Dunmur D A. Small angle X-ray diffraction studies of an ester/biphenyl mixture (5CB/ME 50.5) showing an injected smectic phase. Mol Cryst Liq Cryst Sci Technol. Sect A. Mol Cryst Liq Cryst 258: 239-252 (1995)

[61] Kato T, Kutsuna T, Yabuuchi K, Mizoshita N. Anisotropic self-aggregation of an anthracene derivative: Formation of

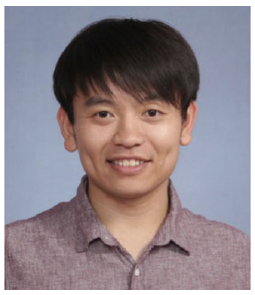

Shaohua ZHANG. He obtained his bachelor and Ph.D. degrees from Tsinghua University in 2008 and 2015, respectively. He works as a design engineer since 2015 at Beijing

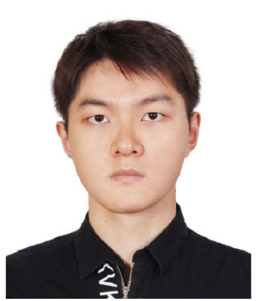

Yijun QIAO. He obtained his bachelor degree in 2015 from Huazhong University of Science and Technology. After then, he was

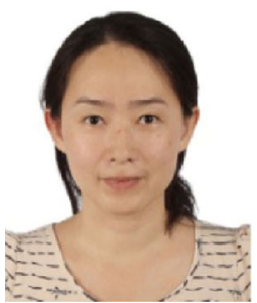

Yuhong LIU. She is an associate professor, received her Ph.D. degree in CAS Key Laboratory of Molecular Nanostructure \& Nanotechnology Institute of Chemistry, Chinese Academy of Sciences, liquid-crystalline physical gels in oriented states. Langmuir 18(18): 7086-7088 (2002)

[62] Arnone A, Cardillo R, Nasini G, de Pava O V, Quaroni S. A new strain of Streptomyces: An anthracycline containing a $C$-glucoside moiety and a chiral decanol. Phytochemistry 27(11): 3611-3617 (1998)

[63] Rudquist P, Dietrich C F, Mark A G, Giesselmann F. Chirality detection using nematic liquid crystal droplets on anisotropic surfaces. Langmuir 32(24): 6140-6147 (2016)

[64] Kishikawa K, Furukawa Y, Watanabe T, Kohri M, Taniguchi $\mathrm{T}$, Kohmoto S. Why chiral tartaric imide derivatives give large helical twisting powers in nematic liquid crystal phases: Substituent-effect approach to investigate intermolecular interactions between dopant and liquid crystalline molecules. Liq Cryst 44(6): 956-968 (2017)

[65] Gao M, Li H, Ma L, Gao Y, Ma L, Luo J. Molecular behaviors in thin film lubrication-Part two: Direct observation of the molecular orientation near the solid surface. Friction, in press, DOI 10.1007/s40544-019-0279-1.

Key Laboratory of Long-life Technology of Precise Rotation and Transmission Mechanisms at Beijing Institute of Control Engineering. His interested research areas include thin film lubrication and tribology of space mechanism.

a Ph.D. student at State Key Laboratory of Tribology of Tsinghua University. His research interests include lubrication mechanism and solid-liquid interfacial structure.

Beijing, China in 2005. She joined the State Key Laboratory of Tribology of Tsinghua University from 2005. Her research areas cover nanotribology, nanostructure, and nanotechnology of surface and interface, chemical-mechanical planarization, and water-based lubrication. 


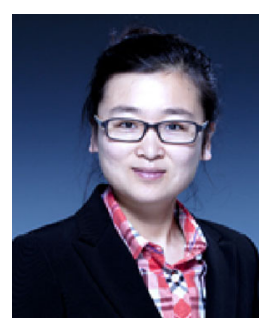

Liran MA. She received her BS degree from Tsinghua University in 2005, and received her Ph.D. degree from Tsinghua University in 2010. Following a postdoctoral period at the Weizmann Institute of Science in Israel, she is now working as an associate professor in State Key Laboratory of Tribology, Tsinghua University. Her interests in

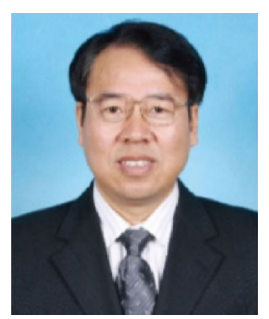

Jianbin LUO. He received his BS degree from Northeastern University in 1982, and got his MS degree from Xi'an University of Architecture and Technology in 1988. In 1994, he received his Ph.D. degree from Tsinghua University and then joined the faculty of Tsinghua University. Prof. Jianbin LUO is an Academician of the Chinese Academy of Sciences and a Yangtze River Scholar Distinguished tribology have ranged from aqueous lubrication and hydration lubrication to the liquid/solid interface properties. She has published over 50 papers. Her work has been cited some 300 times. Her honors include the Hinwin Doctoral Dissertation Award (2011), the Maple leaf award for Outstanding Young Tribologists (2015), and Chang Jiang Scholars ProgramYoung Professor Award (2015).

Professor of Tsinghua University, Beijing, China. He was awarded the STLE International Award (2013), the Chinese National Technology Progress Prize (2008), the Chinese National Natural Science Prize (2001), and the Chinese National Invention Prize (1996). Prof. LUO has been engaged in the research of thin film lubrication, superlubricity and tribology in nanomanufacturing. He was invited as a keynote or plenary speaker for more than 20 times on the international conferences. 